http://dx.doi.org/10.18778/2196-8403.2007.10

MichAEL HAASE

\title{
„Ich vergleiche, also bin ich" - Zur Funktion der Metapher in Hans Magnus Enzensbergers Der Untergang der Titanic
}

Für Herbert Kaiser

Podczas lektury poematu Enzensbergera nieuchronnie narzuca się pytanie o wzajemny stosunek literatury i życia. O ile indeterminacja metafor jest sugestią nieobecności sensu, to leżące u podstaw tekstu autobiograficzne reminiscencje wskazują na zobowiązania znaczeniowe. Autor przedłożonego opracowania podiął próbę mentalnego połączenia obydwu elementów, uznając metaforę pierwotnie za antropologiczną kategorię, która polaryzuje estetyczny dystans oraz egzystencjalny udział. Punktem wyścia do tych przemyśleń jest nieuwzględniane dotychczas przez badaczy źródło, z którego czerpie Enzensberger: esej Osipa Mandelsztama Rozmowa z Dantem (1994a).

Bei der Lektüre von Enzensbergers Poem stellt sich zwangsläufig die Frage nach dem Verhältnis von Schrift und Leben. Suggeriert die Indetermination der Metaphern eine Abwesenheit von Sinn, so verweisen die dem Text zugrundeliegenden autobiographischen Reminiszenzen auf eine Verbindlichkeit von Bedeutung. Der Autor des vorliegenden Aufsatzes versucht beide Momente zusammenzudenken, indem er die Metapher als primär anthropologische Kategorie versteht, welche ästhetische Distanz und existenzielles Betroffensein in einen polaren Zusammenhang stellt. Ausgangspunkt der Überlegungen ist hierbei eine bislang von der Forschung nicht berücksichtigte Quelle Enzensbergers: Ossip Mandelstams Essay Gespräch über Dante (1994a).

Enzensberger's poem inevitably poses questions about the relationship between literature and life. While the ambiguity of the metaphors suggests an absence of meaning, the autobiographical allusions underlying this text point to the necessity of meaning. This essay approaches these aspects of the text as a unity by positing metaphor as a primarily anthropological category that puts aesthetic distance und existential involvement in a polar connection. The starting point of this interpretation is one of Enzensberger's sources that was previously not taken into account: Ossip Mandelstam's essay Conversation about Dante (1994a). 
1. Schon ein Blick auf die Umschlagseite genügt, um zu erkennen, dass bei Hans Magnus Enzensbergers Untergang der Titanic die herkömmliche Navigation des Verstehens versagt. Während der Titel eine Tragödie ankündigt, verweist die Gattungsbezeichnung „Komödie“ genau aufs Gegenteil. Und nach der Lektüre scheint klar, dass sich das Poem einer semantischen Fixierung entzieht. Der Leser wird gleichsam in einen „Strudel von Wörtern“ (ENZENSBERGER 1981:99) ${ }^{1}$ hineingezogen, und der einzige Trost ist, dass es dem Dichter ähnlich geht und er, ,,am Rand des Abgrunds“ stehend, ihm mit mattem Lachen zuruft: „Danke gleichfalls.“ (UT:114) Wo aber bleiben beide, wenn sich die 33 Gesänge und 16 Gedichte unwiderruflich entgrenzen? Ist das Verschwinden einer bedeutungsgebenden Instanz der notwendige Preis für das ,Lust-Spiel` der Sprache? Oder lässt sich zwischen der heiteren Auflösung der Schrift und dem drohenden Untergang von Produzent und Rezipient, zwischen Komödie und Tragödie, ein rettender Mittelweg finden?

Vor diesem grundlegenden Problem steht jede Lektüre des 1978 erschienenen Buches. Schon Hans-Thies Lehmann spricht in dem ersten großen Interpretationsversuch von einem „Meer der literarischen Vorläufer und Vorbilder“ (THIESLEHMANN 1984:318). Folglich kann ein Untergehen im Poem nur verhindert werden, wenn akzeptiert wird, dass der „Eisberg unwiderruflich auf uns zukommt“ (UT:27) und jeder Versuch, sich mit den Mitteln einer letztbegründenden Hermeneutik an Bord zu halten, zum Scheitern verurteilt ist. Nur das Wasser vermag uns zu tragen ${ }^{2}$, da „Bestimmbarkeit unmöglich ist“ ( $\mathrm{KoCH}$ 1997:278), der Sinnfluss dem Verstehen eine Ankunft auf festem Grund versagt. Allerdings besteht kein Zweifel, dass das Poem ,nicht einfach ein dionysischer Text ist." Schließlich weist Enzensberger explizit darauf hin, ,[...] dass das ,Fluten" der ästhetischen Erfahrung auf lebensweltliche Kommunikation bezogen bleiben muss“ (KOCH 1997:291f.). Während das letzte Wort des letzten Gesangs „weiter“ lautet und einen unabschließbaren Verweisungszusammenhang der Schrift suggeriert, ist der darauf folgende entstehungsgeschichtliche Vermerk „La Habana 1969 - Berlin 1977“(UT:115) eine unmissverständliche Markierung eines Anfangs und eines Endes. Demnach besteht zwischen Entgrenzung und Begrenzung ein Spannungsverhältnis. Das freie Spiel mit Bedeutungen läuft auf eine Abwesenheit von Sinn hinaus, während der Autor, indem er einen autobiographischen Bezug herstellt, eine Verbind-

$1 \quad$ Im Folgenden unter der Sigle UT zitiert.

2 „In Enzensbergers Poem ist das Meer nicht nur das Element, in dem man scheitern kann, sondern auch das, das einen weiterschwimmen läßt, also trägt.“ (FETSCHER 2004:286) 
lichkeit von Bedeutung nahe legt. Nur stellt sich die Frage, wie sich beide Sphären miteinander vereinbaren lassen. Um darauf eine Antwort zu finden, gilt es, das Augenmerk auf die Metapher zu richten. Als rein semantisch-semiotische Kategorie steht sie ganz im Zeichen eines referenzlosen Nominalismus. Bedenkt man jedoch ihre anthropologische Struktur, so bietet sich eine andere Perspektive. Wie im sprachlichen Bild die Polaritäten von Geistigem und Sinnlichem, Ideellem und Realem aufeinander bezogen sind, so beim Menschen Natur und Geist, Endlichkeit und Unendlichkeit. Und dieser unhintergehbare Zusammenhang von Sprache und Anthropologie in der Metapher ${ }^{3}$ liefert einen Denkansatz, welcher Tragödie und Komödie, existenzielles Betroffensein und artistische Freiheit verknüpft. Dass Enzensberger diesem Verständnis auch bei der Niederschrift gefolgt ist, zeigt ein verstohlener Hinweis auf eine Quelle, die in der Forschung bislang noch nicht berücksichtigt wurde: Ossip Mandelstams Essay Gespräch über Dante. Anhand dieses Textes und der ihm zugrundeliegenden Maxime „Ich vergleiche, also bin ich.“ (MANDELSTAM 1994a:187) findet sich ein Zugang, bei dem der Leser im Poem auf die drohende Katastrophe im Kopf weder mit titanischer Verstehenswut noch mit ziellosem Hin-und-Herpaddeln reagieren muss, sondern mit dem heiteren Ernst der Aufmerksamkeit.

2. Im Havanna des Jahres 1969, so das lyrische Ich als Alter Ego des Autors, habe er in Kuba geweilt. Getragen von der politischen Aufbruchsstimmung der 68er Revolte verlebte er „die sonderbar leichten Tage der Euphorie“ (UT:15):

Es schien uns, als stünde etwas bevor,

etwas von uns zu Erfindendes.

[...]

Wir suchten etwas, hatten etwas

verloren auf dieser tropischen Insel. Das Gras wuchs

über die abgewrackten Cadillacs. Wo war der Rum,

wo waren die Bananen geblieben? Etwas anderes

hatten wir dort zu suchen - schwer zu sagen,

was es eigentlich war -, doch wir fanden es nicht

in jener winzigen Neuen Welt, wo alles vom Zucker sprach,

von der Befreiung, von einer Zukunft, reich

an Glühbirnen, Milchkühen und nagelneuen Maschinen. (UT:15)

Dieser grundlegende Denkansatz basiert auf den Überlegungen zur „Metapher Mensch“, wie sie Herbert Kaiser seit den 80er Jahren in Beschäftigung mit Jean Paul, Goethe, Lichtenberg, Musil u.a. entwickelt hat. An Texten wären hier - pars pro toto - zu nennen: KAISER (1993); KAISER (1995); KAISER (2005). 
Die Insel war geprägt von der alten Ordnung, die „üppig verblühte“ (UT:14). Dies zeigte sich nicht allein an den zurückgelassenen Relikten jener untergegangenen Welt der Spielcasinos und Striptease-Clubs, in denen sich die aus den USA einreisenden Gangster und US-Senatoren vergnügt hatten, während das Volk Hunger und Not ausgesetzt gewesen war. Die westeuropäischen Intellektuellen, die als ,Revolutionstouristen“ nun die Dinnertafeln der LuxusHerbergen besetzten, genossen ebenso jene Privilegien, die just zehn Jahre zuvor, im Jahre 1959, Fidel Castros Revolte ausgelöst hatten. Jedoch bezeichnet die bei TURGENJEW (1983) entlehnte Formel von den „überflüssige[n] Leute[n]“ (UT:36) nicht nur den parasitären Status der „Pariser Trotzkisten“, die sich im mondänen „Hotel National“ mit Brotkugeln bewarfen, während draußen das Volk geduldig „Schlange um eine Pizza stand“. Es meint zugleich den Überfluss der „Zitate von Engels und Freud“ (UT:36), den prächtigen Überbau, dem jede Verwurzelung in der Materie jener realsozialistischen Wirklichkeit fehlte. Die ,tropische Insel“ $(U T: 15)$ war somit ein Ort des Alten, wo das vermeintlich Neue und Andere, in Gestalt der Trope, des bildlichen Ausdrucks, als unheilkündende Luftspiegelung lediglich die Illusionen des Fortschrittsglaubens offenbarte. Auch der Autor sah den ,tintenschwarze[n], triefnasse[n] Tiefsinn seiner Metaphern“ (UT:53) nicht, als er auf Kuba die Arbeit an seinem Gedicht begann. Denn ,[s]einerzeit glaubte ich jedes Wort, / das ich schrieb, und ich schrieb / am Untergang der Titanic. / Es war ein gutes Gedicht.“ (UT:20) Die „kalte Fata Morgana“ des Eisbergs (UT:17), die er vor Havannas Küste bei einem der endlosen Gespräche erblickte, erkannte er nicht als Wink, dass das utopische Denken dem Untergang geweiht ist: „Ja, / damals sagte ich mir, es ist nur / eine Fata Morgana, in Wirklichkeit, / sagte ich mir, schwankt die Insel Cuba nicht unter unseren Füßen." (UT:21)

Im Berlin des Jahres 1977 findet sich Lichtenbergs Maxime, ,die Metapher ist weit klüger als ihr Verfasser“ (LICHTENBERG 1968:512), beglaubigt. Der ,Deutsche Herbst', welcher mit der Ermordung Hanns Martin Schleyers, dem Geiseldrama von Mogadischu sowie dem kollektiven Selbstmord der RAF-

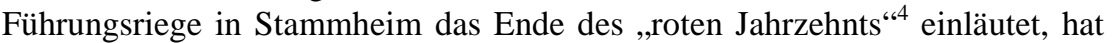
Enzensbergers Glaube an die auktoriale Fixierbarkeit der Tropen zerstört. Sein fertiggestelltes Gedicht vom Untergang der Titanic weiß, dass das Schiff keine Allegorie auf den Kapitalismus sein kann, der in notwendiger Kollision mit den kollektiven Bedürfnissen dem Untergang geweiht ist. Er erkennt, dass schon seinerzeit ,das Fest längst zu Ende, / und alles Übrige eine Sache war / für die Abteilungsleiter der Weltbank und die Genossen von der Staatssicher-

4 So bezeichnete KoENEN (2002) die Jahre von 1967-1977. 
heit, genau wie bei uns und überall.“ (UT:15) Und begleitend zu diesen Molltönen vermerkt er in seinem kleinen Essay Zwei Randbemerkungen zum Weltuntergang, welcher im Jahre 1978 als ein zeitgenössischer Kommentar zum Gedicht erscheint, mit lapidarer Eindringlichkeit,

[...] daß es keinen Weltgeist gibt; daß wir die Gesetze der Geschichte nicht kennen; daß auch der Klassenkampf ein „,naturwüchsiger“ Prozeß ist, den keine Avantgarde bewußt planen und leiten kann; daß die gesellschaftliche wie die natürliche Evolution kein Subjekt kennt und daß sie deshalb unvorhersehbar ist, daß wir mithin, wenn wir politisch handeln, nie das erreichen, was wir uns vorgesetzt haben, sondern etwas ganz anderes, das wir uns nicht einmal vorzustellen vermögen; und daß die Krise aller positiven Utopien eben hierin ihren Grund hat. (ENZENSBERGER 1982:234f.)

Im Lichte dieser Sätze scheint die zeitlich-räumliche Klammer „Havanna 1969 - Berlin 1977“ den Weg zu beschreiben von der Utopie zur Desillusion, von der Hoffnung zur Enttäuschung, von der „Euphorie“ zur Ernüchterung. Aber das veröffentlichte Gedicht vermeidet genau jene Entwicklung. Es ist nicht ausgerichtet auf eine Finalität, es ist weder bestimmt von revolutionärer Ungeduld noch von zynischem Nihilismus. Vielmehr eröffnet die Katastrophe im Kopf des politischen Propheten einen Raum für den Dichter, der den Metaphern weder mit einem totalen Sinnanspruch noch mit einem totalen Sinnverzicht begegnet, sondern das Zwischen besetzt. Und in diesem Zwischen, markiert durch den Bindestrich zwischen den Zeit- und Ortsangaben, liegen die verborgenen Möglichkeiten des „Anachronismus“ (UT:20). ${ }^{5}$ Was in den Au-

$5 \quad$ Koch (1997:279f., 292f.) schreibt: „Der eigentliche Inhalt von Enzensbergers Untergang der Titanic ist - pointiert gesagt - der Bindestrich in den letzten sechs Zeichen des Textes: ,La Habana 1969 - Berlin 1977‘.“ Von dieser Auffassung ausgehend, meint er, das Poem thematisiere „weder das historische Ereignis (April 1912), noch die konkreten Meinungen und Befindlichkeiten jener ,Ichs', die in Berlin und Havanna lebten“, sondern den „Zeitfluß, in dem die Befindlichkeiten und Geschichtsdeutungen sich verändert haben." Konsequenz dieser Veränderung sei die „Auflösung von Modellen wie ,geschichtlicher Fortschritt“ und ,kontinuierliche persönliche Entwicklung “". Jedoch macht Koch deutlich, dass die im Text aufscheinende „Vision eines völligen Endes aller Deutungsanstrengungen und Sinnfestlegungen" von Enzensberger zugleich negiert wird, weil sein Poem bei allem „energetischen Entgrenzen“ von Bedeutung ,auch ein Medium des Überlebens, ja der gesteigerten Rückkehr ins Leben [ist], indem die Kräfte, die durch die Entropie verkrusteten Sinns freigesetzt werden, wieder in eine semiotische Restrukturierung eingehen.“- SusAnNe Komfort-HeIn (2001:318, 335), die auf Koch in ihrer Darlegung zurückgreift, sieht den Bindestrich als „Zwischenraum“ für eine ,artistische Odyssee“, die aber durch ihre „Einbindung in die Di- 
gen des enttäuschten Utopisten nur die Überlebtheit des Glaubens an den Weltgeist benennt, stellt für den Poeten ein taugliches Mittel dar, aus der Linearität der Zeit auszubrechen und über diese zu verfügen „wie ein Gott“ $(U T: 23,26)$. Die faktische „Kälte in meinen Knochen“ (UT:20) im winterlichen Berlin wird so durch ein freies Spiel mit den Tropen - im doppelten Sinne - kompensiert. Demnach verwundert es auch nicht zu erfahren, dass die erste Fassung vom Untergang der Titanic beim Versand von der neuen und in die alte Welt irgendwo „,verschollen“ ist. Das „schwarze Wachstuchheft“ in einem „Kuvert aus braunem Manila-Papier“ (UT:21) ist als Treibgut auf dem Meer unterwegs, eine Not, aus der die zweite Fassung eine Tugend macht, indem sie das lose Treiben in Raum und Zeit zum poetischen Konzept macht, also auf den Verlust (Verlustanzeige, UT:18f.) mit Lust reagiert.

Worin besteht die Lust? Im Abstand, im Abstand zum früheren Ich, zu feststehenden Bedeutungen und Bildern, falschen Gleichsetzungen (von Schreiben und Leben, Theorie und Praxis) und in der dadurch gewonnenen „Aufmerksamkeit" (ENZENSBERGER 1982:236). Der Erste Gesang ist geradezu eine Schule der Aufmerksamkeit. Er zeigt auf, dass der Schreibende nie der Sprechende ist, „die Wörter zu spät kommen, / zu früh“ (UT:61), dass der vorgestellten Identität: „Der da spricht, das bin ich“ (UT:7) die Spur oder der Aufschub der Schrift innewohnt. Aber die Demonstration fußt nicht auf philosophischer Reflexion, sondern auf den Sinnen. Dem Schreibenden ist der Sprechende „ganz in der Nähe, hier“. Und dessen „Ohren“, „diese Antennen aus weichem Fleisch“, relativieren das göttliche Verfügen über das „weiße Rauschen“ (UT:7), indem sie den Aufprall der Titanic auf den Eisberg erlauschen. Die Aufmerksamkeit versagt sich der „Lüge“ der Identität, entzieht sich jedoch zugleich einer falschen Souveränität, indem der Riss im Leib der Titanic das Ich in seiner Leiblichkeit und Verletzbarkeit trifft. Die Distanz des Schreibenden wird so begleitet von der Angst des Sprechenden (,Ein eisiger Fingernagel, / der an der Tür kratzt und stockt.“). Es ist nicht die „Angst vor der eigenen Angst" (ENZENSBERGER 1982:236), welche sich in eine semantische Verbunkerung zurückzieht. Es ist die kreatürliche Angst, welche als Lust dem drohenden Verlust mit vitaler Abwehr begegnet. Insofern ist die ästhetische Revolte gegen das Streben, Unbekanntes auf Bekanntes zurückzuführen, zugleich bemüht, einer Nivellierung von Sinn zu entgehen. Oder wie MAN-

mensionen von Zeit und Raum auf ihr eigenes Begrenztsein und gleichermaßen auf die Unhintergehbarkeit von Sinnstiftung [reflektiert]." - Meine Arbeit teilt diese Ansichten, entfaltet sie aber am anthropologischen Verständnis der Metapher. 
DELSTAM (1994a:227) schreibt: „Die Aufmerksamkeit ist die eigentlich kühne Leistung des Dichters, Zersaustheit und Zerstreutheit sind nur die Ausflüchte einer lyrischen Faulheit.“

3. Mandelstams Diktum ist keine willkürliche Assoziation. Das Zitat findet sich in einem 1985 entstandenen Essay über den tschechischen Collagekünstler Jiří Koláŕ, in dem Enzensberger das Jahr 1968 erinnert und erkennt, dass ,jeder Versuch, den Tumult intelligibel zu machen“, „notwendig im ideologischen Kauderwelsch“ enden musste. Daher konnte die Erinnerung „nur eine Form annehmen: die Collage“ (ENZENSBERGER 1985:8). Durch sie vermochte man den „Lärm, das Stimmengewirr, den Streit, alte und neue Lügen, Wünsche, Hoffnungen, Illusionen“ aus dem „Sechsundvierzigtausend-Bruttoregister-Tonnen-Kopf" (UT:96) der eigenen Erinnerung aufzunehmen, indem man das Gehörte, Gesehene, Erlebte und Erlesene „auftrennte, zerstückelte, zerriß, zerschnitt und kommentarlos verband, aneinanderklebte, neu zusammensetzte." Was hier auf Jiř́ Koláŕ und seine Technik bezogen ist, lässt sich mühelos auf Enzensberger und sein Poem Untergang der Titanic übertragen (KOMFORT-HeIN 2001:331). Wie jener den ,Prager Frühling ' von der anfänglichen Euphorie bis zum Scheitern mit der aufmerksamen „Entschlossenheit, alles zu verarbeiten", verfolgt, ohne damit eine bestimmte Botschaft zu verkünden (ENZENSBERGER 1985:8), so ruft dieser die Geräusche und Bilder aus den Tiefen der eigenen Erinnerung (u. a. an die verlorene erste Fassung) auf und ordnet sie zu einem „Pasticcio“ (UT:54): „Ich restauriere Bilder, ich fälsche mein eigenes Werk." (UT:26) Und genau in diesem Sinne urteilt MANDELSTAM (1994a:166f.) in seinem großen Essay Gespräch über Dante über die ästhetische Methode bei der Niederschrift der Divina Commedia, deren Entstehen er auf den Erfahrungsdruck des Autors zurückführt: „Dante und die Phantasie - das ist doch unvereinbar! [...] Was für eine Phantasie hat er denn? Er schreibt nach Diktat, er ist Kopist, ein Übersetzer. [...] Hier geht es um eine Reinschrift nach dem Diktat der grausamsten und ungeduldigsten Diktierer." Für den russischen Poeten stellt Dante nicht jene von der Rezeption imaginierte mystische Gestalt dar, die ,aus Kapuze und Adlernase bestand und auf irgendwelchen Felsen weiß der Teufel was trieb" (MANDELSTAM 1994a:133). Sein Werk, dessen ,[k]örnige Beimengungen und Lava-Äderchen“ auf eine „Katastrophe als den Ursprung der Formbildung“ (MANDELSTAM 1994a:126f.) hindeuten, ist das „Produkt einer gewaltigen inneren Unausgeglichenheit, die sich einen Ausweg sucht“"(MANDELSTAM 1994a:125). Dass Enzensberger Mandelstams Essay kannte und bei der Arbeit am TitanicPoem als Quelle verwendete, dafür sprechen zahlreiche Anspielungen und Verweise. Die Gattungsbezeichnung, die Anzahl der Gesänge sowie die zahl- 
reichen expliziten und impliziten Auftritte Dantes sind dabei das geringfügigste Indiz. Wichtiger scheint schon Enzensbergers zweite Frau Maria Alexandrowna Makarowa, die den Autor seinerzeit nach Kuba begleitete und im Text mehrere Male als Verschollene auftaucht $(U T: 16,21,111)$. Sie ist als Tochter des Autors Alexander Fadejew ,in der literarischen Szene der ChruschtschowÄra" aufgewachsen, galt als Kennerin der avantgardistischen Literatur der 20er und 30er Jahre und hat Mandelstam selbst übersetzt (LAU 2001:241f.). Zudem erschien dessen Dante-Essay aus dem geretteten Nachlass erstmalig 1967 in Moskau ${ }^{6}$, wo er von den unangepassten Intellektuellen sofort aufmerksam rezipiert wurde. Aber der wichtigste Beleg findet sich an einer höchst markanten Stelle im dritten Gesang. Enzensbergers sprechendes Ich erinnert wieder den Inhalt der endlosen Diskussionen unter den westeuropäischen Intellektuellen auf Kuba:

Wir sprachen in einem Kauderwelsch, Spanisch, Russisch und Deutsch, von der fürchterlichen Zuckerernte der Zehn Millionen, heute natürlich spricht kein Mensch mehr davon. Was geht mich der Zucker an, ich bin Tourist! schrie der Deserteur, dann zitierte er Horkheimer, ausgerechnet Horkheimer in Habana! Wir sprachen auch von Stalin und Dante, ich weiß nicht mehr warum, was hatte Dante mit dem Zucker zu tun. Und ich war zerstreut und blickte hinaus über die Hafenmauer auf die Karibische See, und da sah ich ihn, sehr viel größer und weißer als alles Weiße, weit draußen, ich allein sah ihn und niemand sonst, in der dunklen Bucht, die Nacht war wolkenlos und das Meer schwarz und glatt wie Spiegelglas, da sah ich den Eisberg, unerhört hoch und kalt, wie eine kalte Fata Morgana trieb er langsam, unwiderruflich, weiß, auf mich zu. (UT:16f.)

6 DutLi (2003:553); Dutli vermerkt hier auch, dass es die erste Buch-Publikation des Dichters in seiner Heimat seit 1928 gewesen war. 
Wieso Stalin und Dante in einem Atemzug genannt werden, ist mit Mandelstam leicht zu erläutern, schließlich hatte für den russischen Dichter die Divina Commedia eine genuin politische Dimension. Bereits 1923 nannte er sie das „bedeutendste politische Pamphlet ihrer Zeit“ (MANDELSTAM 1994:216). Und in seiner Vierten Prosa aus dem Jahre 1929/30 findet sich als Zitat der erste Vers aus Dantes Inferno (,Nel mezzo del cammin di nostra vita“; MANDELSTAM 1985:267 ${ }^{7}$, den eigenen Eintritt in die stalinistische Hölle der 30er Jahre andeutend. ${ }^{8}$ Was Dante mit Kubas ,Zucker“ zu tun hat, scheint indes zunächst rätselhaft. Doch die darauf erscheinende „kalte Fata Morgana“ des Eisbergs gibt Aufschluss. In der nahe liegenden Analogie von Zuckerhut und Eisberg wird die „fürchterliche Zuckerernte der Zehn Millionen“, an der Enzensberger als freiwilliger Helfer teilnahm und das erste Mal die Hölle des real existierenden Sozialismus hautnah erlebte, als ein danteskes Inferno kenntlich, geprägt von „Mangel, Korruption und Ineffizienz“ (LAU 2001:255f.). Aus dem Zucker erwuchs gleichsam der Eisberg, an dem die letzten Illusionen zerbrachen. Der Poet Dante ist demnach auch ein politischer Gesprächspartner für Enzensberger, wenngleich der existenzielle Hintergrund bei Mandelstam ein anderer ist. Aber von ihm konnte er lernen, wie das Politische bei Dante zu erfassen ist. Als ,Pamphletist‘ überzeugt dieser nicht durch die stehenden Redewendungen des geschulten Rhetorikers, sondern durch die Kraft der Metapher. Das macht Mandelstam in einer nachgelassenen Notiz zum Essay deutlich, die sich wie ein Programm zur ästhetischen Konzeption des Untergangs der Titanic liest: „Ich vergleiche, also bin ich, hätte Dante sagen können. Er war der Descartes der Metapher. Denn für unser Bewusstsein (und wo ein anderes hernehmen?) offenbart sich die Materie nur über die Metapher, denn es gibt kein Sein außerhalb des Vergleichs, denn das Sein ist selber Vergleich.“ (MANDELSTAM 1994a:187) In diesem Fragment findet sich der Grund für den politischen und existenziellen Raum der Poesie. Gegen die Eindeutigkeit der Systemdenker und Gewissheitssucher, deren Sprache die Struktur des GULAG inhärent ist, stellt Mandelstam den Vergleich als Relativierung des Barbarischen. Zugleich hält er an der Verantwortlichkeit der Poesie fest, indem er

\footnotetext{
In der deutschen Übersetzung von Wilhelm G. Hertz heißt der zitierte Dante-Vers: „Als unseres Lebens Mitte ich erklommen.“(DANTE ALIGHIERI 1978:7)

DUTLI (2003:398); ein Jahr nach der Niederschrift seines Gesprächs über Dante wurde Mandelstam nach Woronesch verbannt und 1938 schließlich in ein sibirisches Gulag deportiert, in dem er wenige Monate darauf starb (DuTLI 2003:418434, 513-528). - In Dutlis aufschlussreicher Darstellung findet sich auch der Hinweis auf die oben angeführten Zitate.
} 
ihren Offenbarungscharakter betont und damit ihre Verbindlichkeit. Wirklichkeit ist präsent im Vergleich, da das Sein selbst Vergleich ist. Der Ursprung ist immer schon Zitat, das weiß der aus Florenz verbannte Dichter ebenso wie der aus Moskau verbannte Mandelstam, jedoch offenbaren sich in diesen Zitaten, Vergleichen, Übertragungen: Leid, Trauer, Angst, Hass, also die konkreten Gefühle dessen, der da schreibt. Das weiß auch Enzensbergers ,Bauchredner ‘ im Titanic-Gedicht. Nach dem Schiffbruch der Utopie lässt er sich als Schwimmer von einer Welle zur nächsten übertragen, aber heult dabei (UT:115).

4. In Mandelstam findet sich ein vorzüglicher Führer durch das Enzensbergersche Poem. Wie Vergil einst den italienischen Autor durch die Höllenkreise geleitete, so vermag uns der Dante-Essay des russischen Dichters durch die Kreise $^{9}$, die der Untergang der Titanic auf der Wasser-Oberfläche hinterlassen hat, zu führen. Er reicht uns den „Faden“ (UT:22, 109), welchen das Ich im Poem verloren hat und beständig sucht. Der erste Anknüpfungspunkt dafür ist Dantes Person. Der zwischen dem 23. und 24. Gesang eingefügte Abschnitt Erkennungsdienstliche Behandlung (UT:78) stellt einen Bedeutungsfächer dar, der, ausgehend von der festgestellten Nichtidentität (,Das ist nicht Dante.“) bis zu seinem Gegenteil („Das ist Dante.“), alle Spielarten der Differenz durchläuft, bis er in der Erkenntnis mündet, dass die Identität im Unsagbaren liegt, derjenige Dante ist, „,den niemand für Dante hält außer Dante.“ Jedoch folgt diese poststrukturalistische Herleitung einer ,armseligen logischen Notwendigkeit“, die der „Kraft des Danteschen Vergleichs“ (MANDELSTAM 1994a:131f.) kaum gerecht wird. Denn bei ihm - wie Mandelstam anmerkt - bedeutet die Sprache ,immer unterwegs zu sein“ (MANDELSTAM 1994a:127). Die Reise ins eigene Ich, welche als Motiv der Divina Commedia ebenso zugrunde liegt wie dem Untergang der Titanic, verläuft als Textbewegung nicht linear, vom titanischen Willen nach absoluter Selbsterkenntnis getrieben, sondern gleicht dem Segeln, „diese[m] geschmeidigsten und plastischsten, der Menschheit seit Urzeiten bekannten Sport" (MANDELSTAM 1994a:141). So sagt es der Autor des Dante-Essays und liefert damit eine Einsicht, die ENZENSBERGER (1989:305) in seinen Vermutungen über die Turbulenz so ausdrückt: „Die Frage, ob es mit dem Strom oder gegen ihn zu schwimmen gilt, scheint mir veraltet, weil sie eine unerträgliche Vereinfachung voraussetzt. Ergiebiger scheint mir das Verfahren des Seglers zu sein, der sowohl mit dem Wind als auch gegen ihn kreuzt." Was hier als pragmatischer Ratschlag für kritische

9 Thies-Lehmann (1984:316) spricht von einer „kreisenden Anordnung“ der Gesänge und Gedichte im Untergang der Titanic. 
Intellektuelle daherkommt, liefert dem Autor der zweiten Fassung des Untergangs der Titanic eine poetische Strategie, welche der persönlichen Katastrophe nahe kommt, ohne ihr ausgeliefert zu sein. Anders als die falsche Alternative „Titanic“ oder „Untergang“ ist sie „plastisch“, weil ein Verstehen des in die geschichtliche Welt gestellten Ich nicht auf Bedeutungen verzichten kann, und zugleich „geschmeidig“, als sie sich mit den Relativierungen des Ästhetischen dem Dogma der Identität entzieht. Zwischen Fiktion und Authentizität gestellt, ähnelt sie dem Traum, der als Leistung der Einbildungskraft stets auf Erfahrungen gründet, also einen „Eigensinn“ anzeigt. Nichts anderes meint der 9. Vers der Erkennungsdienstlichen Behandlung, welcher genau ihre Mitte markiert: „Das ist ein Mann, der träumt, er sei Dante.“ (UT:78) Das Segeln, welches an den Kreuzungspunkten von Ich und Geschichte agiert, ermöglicht es, die Wellenkreise anzusteuern. Der Untergang der Titanic ist gleichwohl „,aktenkundig“, aber ,auch nicht mehr das, was er einmal war“ (UT:55). Nicht nur Erinnerungen, Zeugenberichte, Verfilmungen (UT:37, 89f.) haben das ursprüngliche Ereignis längst bis zur Unkenntlichkeit entstellt, sondern als Mythos hat es bereits einige Metaphorisierungen durchlaufen, weshalb es sinnlos anmutet, gegen deren Wogen zu steuern. Fruchtbarer scheint eine Darstellung, welche „eigentümliche Selbstbekenntnisse“ (MANDELSTAM 1994a:155) in den Strom der Überlieferung einbringt und so Schnittstellen findet, an denen der intendierte Eigen-Sinn des Schiffbruchs kenntlich wird. Wie Dante dem Diktat der Tradition folgt, aber aus der Rolle des simplen Chronisten durch das Selbstdiktat heraustritt und die Hölle mit nahen und fernen Zeitgenossen bevölkert, so sind Enzensbergers Worte wie Segelschiffe, die den Strömungen folgen und auf Signalwellen des Sinns lauern, um diese zu kreuzen. Die dadurch entstehenden „Vergleiche“ stellen als „,artikulierte Ausbrüche“ (MANDELSTAM 1994a:173) einen Zusammenhang her zwischen alter und neuer Welt und offenbaren so die ewige Crux allen utopischapokalyptischen Denkens: dessen unversiegbaren Anspruch auf Gewissheit.

Wenn ein Ingenieur der Titanic als klassischer Homo faber mit Verweisen auf die Statistik der Schiffbrüche und die Gesetze der Evolution „den eigenen Tod" als bedauerlichen Einzelfall beschreibt (UT:34f.), so gleicht er Friedrich Engels, der im Disput mit Bakunin an Bord des sinkenden Dampfers dessen anarchistischen Thesen „die Vorzüge strikter Disziplin“ entgegenhält (UT:42f.). Und der russische Sozialrevolutionär wiederum erkennt nicht, dass die armen Auswanderer im Zwischendeck ,ganz andere Hoffnungen“ (UT:25) hegen und von einem spontanen Aufstand ebenso wenig zu überzeugen sind wie der Heizer Shine, der sich den Befehlen des „Käptens“ widersetzt und auch dessen Tochter und ihren eindeutigen Angeboten trotzt (UT:65f.). Die Innere 
Sicherheit (UT:39-41) des Bewusstseins scheitert am Eigenwillen der Materie, was freilich nichts an der Erfahrungs-Resistenz der Fortschrittsgläubigen ändert. Immer wieder schließt sich der Deckel ihrer Kiste ,aus Sicherheitsgründen" (UT:41), kann sie die Dialektik von Utopie und Apokalypse weder „,erschüttern“ noch „,verbittern“ (UT:48f.). Mit der Losung „Nur die Ruhe“ (UT:69f.) reagieren sie auf den ausbleibenden Untergang des Bestehenden ebenso „unverzagt“ (UT:70), wie sie den drohenden eigenen ignorieren. So „packen“ die Philosophen auf dem Hegel-Kongress (UT:93f.) im „verbunkerten Seminar“ ihre „Kristallkugeln und Horoskope aus“, ohne sich von der Einsicht, dass Hegel mit Schnurrbart wie Stalin aussieht, erschrecken zu lassen. Die „Propheten mit dem Rücken zum Meer“ (UT:87f.) erweisen sich als unbelehrbar. Als riesige Forschungsgemeinschaft von Hirntieren ist ihnen die unbestreitbare Tatsache, dass ,wer arm ist, schneller unter[geht]“ (UT:71), gleichsam „ein süßer Trost bei trüber Aussicht“ (UT:70), da so beim ewigen „Ping-Pong die Fackel nicht ausgeh[t]“ (UT:88).

Die zahllosen „Arien und Ariosos“ (MANDELSTAM 1994a:155), mit denen Enzensberger im Poem einen immer währenden Kreislauf der „Wiederholung“ (UT:103) nachzeichnet, relativieren nicht nur die eigenen „Gesänge“, indem sie jede Gefahr einer pathetischen Selbststilisierung abwehren und die Tragödie in ein Spiel verwandeln. Sie haben auch den Effekt, dass der Untergang, wie der Mittelvers des 16. Gesangs verlautbart, als aktenkundige Tatsache und Mythos „den Weg in eine bessere Zukunft [eröffnet]“ (UT:55). Denn wo immer neue „Flaschenposten“10 aus der Tiefe hervortauchen und „kein Ende des Endes" abzusehen ist (UT:98), erweist sich jeder Glaube an den Untergang als Komödie. Das drücken auch die geradezu appellativen Worte im 29. Gesang aus, die im direkten Aufruf an die Zeitgenossen fordern: „Hören wir endlich auf, mit dem Ende zu rechnen! Wer glaubt schon daran, daß er dran glauben muß?“ (UT:99) Die „,bessere Zukunft“ ist nicht mehr auf ein Endziel ausgerichtet, sondern im Bild der immer wieder hervortauchenden „Flaschenposten“ wird klar, dass das „Dinner immer weiter“ geht, dem Menu der Apokalypse stets ein neuer Gang folgt und die Differenz zwischen Untergangsapostel und tatsächlich Untergehenden so grundsätzlich ist wie der „Unterschied zwischen

10 Auch das Bild der Flaschenpost geht auf Mandelstam zurück (MANDELSTAM 1994:9). Allerdings hat es in der Dichtung des 20. Jahrhunderts bereits weite Kreise gezogen, wie z.B. eine zentrale poetologische Selbstaussage des Mandelstam-Übersetzers Paul Celan (1968:128) belegt. Daher ist bei der Annahme, es handele sich in Enzensbergers Poem um ein direktes Zitat, durchaus Vorsicht geboten. 
Leben und Tod" (UT:99). Hier liegt auch die Erkenntnis, zu welcher Dante unterm „Diktat der grausamsten und ungeduldigsten Diktierer“ gelangt sein muss. „Mit zunehmender Länge“, so MANDELSTAM (1994a:126) in seinem Essay, ,entfernt sich das Poem [die Divina Commedia - M. H.] von seinem Ende, und das Ende selbst kommt unerwartet und klingt wie ein Anfang." Die „bessere Zukunft" zeichnet sich also wesentlich dadurch aus, dass sie offen ist, der Blick in sie von der Angst vor dem Tod genauso bestimmt wie von der Lust am Leben. Es ist ein Innehalten, von welchem auch die Metapher als Medium des Vergleichs geleitet ist. Mit ihrem Anteil an Grenze und Grenzenlosigkeit bewirkt sie ein „Stehenbleiben der Zeit“ (MANDELSTAM 1994a:171) im teleologischen Sinne. Zwischen Inferno und Paradies navigierend ist die Metapher als sprachliche Form ein direkter Ausdruck der Conditio humana, ein Schweben zwischen geistiger Freiheit und leiblich-sozialer Bedingtheit.

5. Es gibt in Enzensbergers Titanic-Poem vier Gedichte, welche sich auf erfundene und reale Gemälde beziehen und in denen sich gleichsam die Quadratur des Kreises, das im logischen Sinne Unmögliche, welches die Metapher vermag, zeigt. In einer stufenförmigen Annäherung an das Problem, welche zugleich das Zyklische des Fortschritts in der Kunst vor Augen führt, wird die Präsenz von Bedeutung und damit der existenzielle Ernst von Bildlichkeit in der Kunst erfragt. Das erste Beispiel: Apokalypse, Umbrisch, etwa 1490, führt uns ins Italien der Hochrenaissance. Ein Maler, der sich dem Namen nach nicht identifizieren lässt, erhält von einem ,geizigen Karmeliter“ (UT:12) den Auftrag für eine bildliche Darstellung des biblischen Weltuntergangs. Im Kontext von Enzensbergers Poem folgt der Text dem zweiten Gesang, der den Aufprall der Titanic am Eisberg schildert (UT:10f.) und aus produktionsästhetischer Perspektive die große Herausforderung des Beginns thematisiert, welche im ersten Gesang bereits im Mittelpunkt stand: „Wie fängt man es an, / den Weltuntergang zu malen?" (UT:12) Beide Situationen, die am Schreibtisch und im Atelier, eint ihr Anachronismus. Doch während der Dichter den Unterschied zwischen der Katastrophe und ihrer Darstellung reflektiert, grübelt der Maler lediglich über „technische Fragen“ und „Kompositionsprobleme“ nach. Bei Wintereinbruch in seinem ruhigen und von einem Kamin beheizten Atelier sitzend, ist ihm allein bewusst, dass ,die ganze Welt zu zerstören viel Arbeit [macht]“: „Alles / nämlich soll zerreißen, zerrissen werden, nur nicht die Leinwand.“ (UT:12) Das „Bild“ steht hier, ohne Stellvertreterschaften und differenzierende Verweisungszusammenhänge, unmittelbar dem Glauben an das göttliche Prinzip gegenüber, das als eine metaphysische Instanz einen letztbegründenden Ursprung und damit die Anwesenheit von Bedeutung garantiert. Und so vollzieht sich die Entstehung des Gemäldes scheinbar ganz organisch. 
Der zyklische Wechsel der Jahreszeiten vom Winter über den Frühling und Sommer bis in den Herbst hinein korrespondiert direkt mit dem Kirchenkalender (von Weihnachten über „Mariä Lichtmeß“ bis hin zu „Allerseelen“, $U T: 12)$. Der Künstler agiert völlig ohne Eigensinn, folgt in den Details strikt den biblischen Vorgaben ${ }^{11}$, weshalb er, als der „Weltuntergang glücklich vollendet ist“, sich „unsinnig heiter, wie ein Kind“(UT:13) fühlt. Die Apokalypse als Kunstwerk wirkt auf ihn belebend, aber weniger in einem selbstoffenbarenden als in einem selbstvergessenen Sinne.

In dem zweiten Beispiel: Abendmahl. Venezianisch, 16. Jahrhundert, spielt auch das ästhetische Vergnügen an der Darstellung der bevorstehenden Katastrophe eine Rolle, allerdings ist hier der Künstler nicht mehr der verlängerte Arm göttlicher Notwendigkeit, sondern zeigt ein ganz deutliches Bestreben nach Emanzipation. Ausdruck dieser Individualität ist nicht zuletzt die Tatsache, dass der Maler des Bildes, welches nach dem Streit mit den „Sieben Richter[n] der Inquisition“ (UT:31) schließlich unter dem Titel „Ein Dîner bei Herrn Levi“ an die Öffentlichkeit gelangt, namentlich zu benennen ist. Es handelt sich um Paolo Veronese, der bei der Gestaltung biblischer Themen auch den „Welthorizont“" Venedigs zeigte, ,[...] des ersten großen, ganz modernen Staatswesens, in dem es kein herzogliches oder großherzogliches oder gar päpstliches Reglement des Geistes und Geschmackes gab“ (BADT 1981:19). Insofern ist seine Abendmahlsdarstellung ganz eingebunden in das erwachende Selbstbewusstsein der Zeit. Sein Stil, das relativ milde Vorgehen der kirchlichen Zensoren in der Seefahrer- und Handelsmetropole nutzend, löst sich von den strikten inhaltlichen Vorgaben. Nicht mehr allein Geschöpf, sondern auch Schöpfer, bringt Veronese seine eigene Phantasie ganz gezielt ins Bild ein, versieht dieses spontan mit lebensweltlichen Details, phantastischen Architekturen und stellt marginale bzw. frei erfundene Gestalten ins szenische Geschehen. Mit diesen Eigenwilligkeiten verbindet sich freilich keine versteckte Kritik am Klerus und seiner Macht, seine Leerstellen der Bedeutung sind kein Ausdruck ketzerischen Zweifels. Auf die Einwände der Inquisition antwortet er: „Meine Herrn, sprach ich, dies alles / habe ich frei erfunden zu meinem Vergnügen." (UT:31) Das ästhetische Amüsement ist für ihn die grundlegende Voraussetzung für das Entstehen von Kunst, eine Herausforderung des Sinn-

11 DelisLe (2001:195), von deren akribischer Untersuchung der vier Bildgedichte auch meine Arbeit profitiert, schreibt: „Die geistige Nähe des Malers ist an Ausdrücken zu erkennen, wie ,das Zerreißen des Vorhangs im Tempel', das in Matthäus 27 (51f.) steht, oder , die entflohenen Inseln', die originales Lutherdeutsch sind." 
glaubens ist von ihm nicht intendiert, sondern ein lästiger Nebeneffekt. In seiner Heiligen Anna selbdritt übermalt er sogar wieder "die unter den Thron“ gesetzte „Suppenschildkröte“, weil er das „Rülpsen der Besserwisser“, das nervende "Gebrabbel“ der bestallten Exegeten vorausahnt (UT:33). Diese von Enzensberger erfundene Episode rückt den Künstler Veronese in die Nähe einer ,l'art pour l'art'-Ästhetik, ohne ihn damit zu denunzieren. Denn der Autor und sein lyrisches Ich wissen aus eigener leidvoller Erfahrung um die Verstehenswut der Interpreten. Im 15. Gesang wird letzteres von ihnen einem ähnlichen Verhör unterzogen wie einst der venezianische Maler. Und mit ähnlicher Verachtung reagiert es auf ihre skeptischen Fragen: „Ihr seid [...], /die jeden Hauch aufgabeln, alles zermanscht ihr und raspelt / Bedeutungen mit dem Tranchiermesser runter -“(UT:53). Nur ist die Identifikation mit Veronese gleichzeitig begleitet vom Bewusstsein der Differenz. Die Freiheit der alten Meister in der italienischen Malerei ist abhängig von der Macht der Kirche, denn dank deren Einfluss und Deutungshoheit ist die Präsenz der biblischen Themen eine gegenwärtige. Ein ahistorisches Kopieren seiner Haltung würde daher zwangsläufig zu einem Verfall des Ästhetischen führen, weil die Säkularisierung und damit verbundene Profanisierung religiöser Inhalte dem bloßen Amüsement den Hauch des Banalen verleiht.

Dies zeigt deutlich das dritte Bildgedicht, welches den Titel trägt: Der Raub der Suleika. Niederländisch, Ende des 19. Jahrhunderts. Der Titel ist schon per se beredt, er vermischt biblisch-orientalische und antike Zitate, bedient sich dieser völlig frei, ohne ihre tradierte Bedeutung zu achten. „Der Raub der Helena" wird übertragen auf Suleika, die Gattin des impotenten Hofbeamten Potiphar, welche Jussuf, den biblischen Joseph, hoffnungslos begehrt ${ }^{12}$, und aus beiden Motiven entsteht eine neue Geschichte, die in ihren Motiven an Schöpfungen von Karl May gemahnt. ${ }^{13}$ Niederländisch ist allein der Kunstfälscher Salomon Pollock, den Enzensberger als Salonmaler der Titanic in seinem

12 Die biblische Geschichte von Jussuph und Suleika ist in der orientalischen Literatur weiterentwickelt bzw. variiert worden, aber von einem Raub ist dort nirgends die Rede. Vor allem Goethe bediente sich dieser Vorlagen, als er am West-östlichen Divan arbeitete; vgl. GoETHE (1981:27, Musterbilder, 1981:62-88, Buch der Suleika, und 1981:593, Anmerkungsteil).

13 Bei May zählte die freie Entlehnung von Motiven aus dem Fundus der Geschichten aus 1001 Nacht zur Schreibpraxis. Interessant ist hier besonders der Roman Scepter und Hammer (1879), in dem sich die beiden Motive der Enthauptung und des vergifteten Buchs, welche Pollock erwähnt, finden (SCHWEIKERT 1985:3-7). Bemerkenswert scheint auch die Tatsache, dass May just im Jahre 1912 gestorben ist. 
Poem auftreten lässt. Für Pollock ist das Suleika-Bild ,mit einem aus der Luft gegriffenen Orient“ (UT:84) das Werk, welches er ,nie aus den Augen verliert" (UT:81), weil er es als die Krönung seines Lebenswerkes betrachtet. Als Meister der Fälschung kennt er alle Kniffe und Tricks der ,alten Meister“, im Rijksmuseum sind sogar einige seiner ",heiligen Pfuscherei[en]“ (UT:82) als vermeintliche Originale zu bewundern. ${ }^{14}$ Aber das perfekte Kopieren vereitelt zugleich den Versuch, ,"selber" zu malen“. Jeder Anlauf scheitert, denn das Ergebnis sei stets „,schlecht“ (UT:84). Pollock, der seine ästhetische Impotenz mit Alkohol betäubt, ist ein typischer Vertreter des Historismus, der Ende des 19. Jahrhunderts seine Hochphase erlebt. Die Malerei dieser Kunst-Strömung man denke nur an den Wiener Maler Makart - zeichnet sich durch eine vollständige Immanenz der verwendeten Symbole und Formen aus. Da das prinzipiell historische Denken besagt: alles ist bereits gewesen, gedacht, gemalt, geschrieben worden, gibt es keinen Eigensinn mehr. „Die Wahrheit ist stumm“ (UT:84), wie Pollock weiß, als „Reue der Nachwelt, die unvollkommen ist / und keine Erlösung kennt" (UT:83). Auch in dieser resignativen Einsicht weiß sich der Autor des Titanic-Poems dem Maler durchaus verwandt. Sein Gedicht zehrt bekanntlich von der Erkenntnis, dass sein Untergang kein vollkommener ist und der erlösende Neuanfang nie eintreten wird. „,In Wirklichkeit ist nichts geschehen. “, diese Formel führt er im 27. Gesang gleich bewusst als Zitat an, um den Schiffbruch als „Film“, „Omen“ und „Halluzination“ (UT:91) zu bezeichnen, ist doch alles beim Alten geblieben und selbst die Dichter sitzen noch im Bordcafé, mit schlechtem Gewissen ,der Gastarbeiter, der Eskimos, und der Palästinenser im Zwischendeck“ (UT:92) gedenkend. Im ,überschwemmten Kopf" (UT:96) zerfließen die Bilder ebenso wie bei Pollock, erweist sich „die Geschichte / mit ihren endlosen Finten und Künsten“ als „ein ewiges Hin und Her: / Sie erfindet mich, ich erfinde sie.“ (UT:84) Nur liegt in dieser Erkenntnis, als Schöpfer immer auch Geschöpf zu sein, eine Denkmöglichkeit verborgen, die auf die Metapher verweist. Den alten Gott der Metaphysik gibt es ebenso wenig wie den Weltgeist, insofern ist die Immanenz des Zeichens als Spur und Aufschub zu denken, die eine reale Präsenz nicht ermöglicht. Die Bedeutung befindet sich stets auf der Flucht. Gleichzeitig bleibt jedes menschliche Denken auf sie bezogen, da ,,[... nur göttliche, nicht-endli-

14 Pollocks Behauptung, einige seiner Bilder hingen als Originale im Rijksmuseum, erinnert an den berühmten Fälscher Han van Meegeren, der in den 30er und 40er Jahren des 20. Jahrhunderts mehrere Gemälde schuf und als Werke von Vermeer und Pieter de Hooch erfolgreich an staatliche Museen verkaufen konnte, wo sie bis zu seiner Enttarnung auch ausgestellt wurden (GoLL 1962:179-187). 
che Geister auf Bedeutungen: auf Festlegungen im unendlichen Lesen der Welt zu verzichten [vermögen]. Denn Leben verlangt Handeln, verlangt Entscheidung, Ende und Anfang.“ (KAISER 1993a:183) Insofern ist die Metapher als Zwischen von Identität und Differenz eine genuin anthropologische Kategorie ganz im Sinne von Mandelstams Diktum: „Ich vergleiche, also bin ich.“ Und in dieser Anbindung des Übertragens an die humane Existenz in ihrer Bedingtheit liegt die Verbindlichkeit des Flüchtigen.

Nichts anderes führt das letzte Bildgedicht vor Augen: Ruhe auf der Flucht, Flämisch, 1521. Der neutestamentarische Topos scheint zunächst nur ein blindes Zitat im Sinne Pollocks zu sein, denn von der Flucht der Heiligen Familie nach Ägypten (Matthäus 2, 13-15) ist an keiner Stelle die Rede. Vielmehr widmet sich ein betrachtendes Ich den vielen marginalen lebensweltlichen Details im Bilde, welche die Idylle und Katastrophe des biblischen Themas vergleichend widerspiegeln. Dies entspricht ganz dem niederen Stil (Sermo humilis) der flämisch-niederländischen Malerei, der im Gegensatz zum hohen Stil (Sermo sublimis) der italienischen Renaissance das Offenbarwerden Gottes im Menschen Christus im gemeinen bürgerlichen Leben ansiedelt. ${ }^{15}$ So zeigt sich die Doppelnatur Christi als Metapher für die Polarität des Menschen als Körper und Geist, wodurch im Bild Schrift und Leben im gleichnishaften Sinn aufeinander bezogen werden. Dies widerfährt auch dem lyrischen Ich des Gedichts, welches zunächst aus einer göttlichen Perspektive das Zusammenspiel von Idylle und Katastrophe betrachtet. Es sieht das spielende Kind, welches den Kampf des Bauern mit dem Bären, in dessen Rücken ein Messer steckt, nicht wahrnimmt; den Spielmann, der achtlos an den Gebeinen eines Geräderten vorbeigeht, und die beiden kämpfenden Heere, über denen schon der Sperber kreist. Die Gleichzeitigkeit der Ruhe, die sich, bedrängt von Gewalt und Tod, auf der Flucht befindet, und die Ruhe inmitten der Flucht bleibt ihm „,undurchdringlich“ und „lautlos“, und so wendet es sich ab und lebt weiter. Aber das schreibende Ich, welches das betrachtende Ich ist und durch die Differenz zwischen Betrachten und Schreiben zugleich nicht ist, beschließt das Gedicht mit den Worten: „Ich habe dies alles gesehen, nur / das Messer, das mir im Rücken steckt, nicht.“ (UT:101) Hier zeigt sich die unhintergehbare Polarität von Distanz und Betroffensein, von geistiger Freiheit und leiblicher Bedingtheit sowie von Wahrheit und Lüge. Die Dichter lügen, wenn sie suggerieren, das Dunkel des gelebten Augenblicks sei identisch mit der Schrift. Doch vermag diese durch Metaphorisierung die Idylle in der Katastrophe und die Katastrophe in der Idylle zu offenbaren. Und so entsteht genau jener Ver-

15 KAISER (1995:129-145; 2. Ausschweifung: Poesie und Offenbarung). 
fremdungseffekt, den BRECHT (1993:271) ${ }^{16}$ in den Bildern des niederländischen Malers Pieter Breughel entdeckte: „Wenn der Breughel seine Gegensätze auch ins Gleichgewicht bringt, so gleicht er sie doch niemals einander an. Noch gibt es bei ihm eine Trennung des Tragischen vom Komischen, sondern sein Tragisches enthält selber Komisches und seine Komik Tragisches." Genau dies tut Enzensberger in seinem Titanic-Poem. Wie er als Chronist die „unvorstellbare Ruhe" auf der Titanic beim Gang in die Rettungsboote (UT:47) und die „Schreie“, nachdem das Schiff „lotrecht" in den Fluten verschwunden ist (UT:56), in einen tragikomischen Bezug bringt, so stellt er in seiner Selbstreflexion als Autor gegen die Alternative „Gleichsetzung“ oder „Trennung“ das "Gleichgewicht": von Schrift und Leben, von Geist und Natur. Denn dem Schwebezustand des Vergleichs ist die Weisheit der Ungewissheit eigen und damit jener Eigensinn, der Ruhe und Aufmerksamkeit in seiner unauflöslichen Verklammerung denkt.

6. Wenn MANDELSTAM (1994a:173) anmerkt, die Vergleiche als ,artikulierte Ausbrüche“ hätten Dante zu „dem größten Meister und Gestalter der , poetischen Materie““ gemacht, zum „größten Dirigenten der europäischen Kunst, der die Formierung des Orchesters um Jahrhunderte vorweggenommen" habe, so weiß er, dass diese Leistung nicht zuletzt in den „Besonderheiten der Danteschen Psyche“ wurzelte, seiner „Angst vor direkten Antworten, die vielleicht durch die politische Situation jenes höchst gefährlichen, verworrenen und räuberischen Jahrhunderts bedingt war" (MANDELSTAM 1994b:141). Gewiss wiegt die gleichnishafte Bedeutung dieser Worte schwerer, wenn man auf das stalinistische Inferno im Moskau des Jahres 1933 schaut statt auf die Illusionen der westdeutschen Linken 33 Jahre nach Ende des Zweiten Weltkrieges. ${ }^{17}$ Aber die existenzielle Notwendigkeit eines anthropologischen Verständnisses der Metapher ist von der gleichen Dringlichkeit. Die Katastrophe im Kopf lässt sich weder durch blinde Angst noch durch blinde Hoffnung besiegen, der

16 GRIMM (1984:158) hat auf Brecht als mögliche Quelle für das ,flämische Bildgedicht" verwiesen. Für einen weiterführenden Vergleich interessant ist auch Enzensbergers Essay Das flämische Fenster. Ein Vexierbild (ENZENSBERGER 1989:310-317). FETSCHER (2004:272) sieht in ihm einen „Schlüsseltext“ für das Verstehen der „Gemäldegedichte“.

17 Nicht nur die Zahl der Gesänge entspricht der Anzahl der Jahre, die das Erscheinen des Untergangs der Titanic (1978) vom Jahre 1945 trennen. Addiert man die 16 Gedichte noch hinzu, so verweist die Gesamtsumme der Texte (49) auf das Lebensalter des Autors bei Veröffentlichung des Poems - ein weiterer Beleg dafür, dass beim ästhetischen Spiel mit Verweisungszusammenhängen die Referenz auf den Autor als Betroffenen nie fehlt. 
kreatürliche Schrei bleibt ebenso abstrakt wie das Begriffsgebäude des Weltgeists. Nur die Mitte bringt Vermittlung, macht Sprache zum Gefäß von Erfahrung, Schrift als Leben lesbar. Die Metapher, durch die „Literatur nicht als ein fertiges Produkt erlebt“ wird, sondern als „,kalligraphische Komposition“, vereint Stimme und Wort und sichert dessen Status als "Lockspeise des Sinns“ (MANDELSTAM 1994a:168). Einerseits ,vorläufig nur [...], doch ohne Panik“ (Der Aufschub, UT:44) und andererseits ,,jetzt, wo es zu spät ist“" (UT:67) bietet die Metapher für die Träumer des Absoluten nur einen ,schwachen Trost“ (UT:57), aber in ihrer „englischen Sanftmut“ (UT:58), also ihrem Humor, zeigt sie, dass der Untergang immer schon stattgefunden hat (,wann: ,damals'? 1912? 18? 45? 68?", UT:97) und immer wieder stattfinden wird, was beruhigend und beunruhigend zugleich ist, zum Weinen wie zum Lachen. Ein Erkenntnistheoretisches Modell, welches sich dieser Einsicht verweigert, indem es nach dem Ursprung strebt, mündet zwangsläufig in „vollkommene Leere“ (UT:73f.), der Vergleich hingegen lehrt, was Sprache von Anfang an gewesen ist: „Erinnerung an die Zukunft.“18

\section{Literatur}

BADT, KURT (1981): Paolo Veronese. Köln.

BRECHT, BERTOLT (1993): Verfremdungstechnik in den erzählenden Bildern des älteren Breughel. In: BRECHT, BERTOLT: Werke. Bd. 22.1. Hrsg. von Werner Hecht, Jan Knopf et alii. Frankfurt (M.) / Berlin, 270f.

Celan, Paul (1968): Ausgewählte Gedichte. Zwei Reden. Frankfurt (M.).

DANTE Alighieri (1978): Die Göttliche Komödie. Aus dem Italienischen von Wilhelm G. Hertz. München.

DELISLE, MANON (2001): Weltuntergang ohne Ende. Ikonographie und Inszenierung der Katastrophe bei Christa Wolf, Peter Weiss und Hans Magnus Enzensberger. Würzburg.

DutLI, RalPh (2003): Mandelstam. Meine Zeit, mein Tier. Eine Biographie. Zürich.

EnZensberger, Hans Magnus (1981): Der Untergang der Titanic. Eine Komödie. Frankfurt (M.).

- (1982): Politische Brosamen. Frankfurt (M.).

- (1985): Erinnerungen an einen Tumult. Zu einem Tagebuch aus dem Jahre 1968. In: ARnOld, HeInZ Ludwig (ed.): Hans Magnus Enzensberger. München (=text+kritik 49), 6-9.

18 So lautet der Titel einer Werkauswahl Enzensbergers, die 1988 bei Reclam-Leipzig erschienen ist (ENZENSBERGER 1988). 
Michael Haase

- (1988): Erinnerung an die Zukunft. Poesie und Poetik. Hrsg. von Dorothea Dornhoff. Leipzig.

- (1989): Der Fliegende Robert. Gedichte, Szenen, Essays. Frankfurt (M.).

FETSCHER, Justus (2004): „Der Untergang der Titanic“ und das Überleben der Literatur. Enzensbergers poetische Elementarkunde. In: GOEBEL, ECKART / LÄMMERT, EBERHARD (eds.): „Für viele stehen, indem man für sich steht“. Formen literarischer Selbstbehauptung in der Moderne. Berlin, 260-289.

Goethe, Johann WolfGang (1981): Werke. Bd. 2: Gedichte und Epen II. Hrsg. von Erich Trunz. München.

Goll, JOACHIM (1962): Kunstfälscher. Leipzig.

GRIMM, ReINHARD (1984): Das Messer im Rücken. Utopisch-dystopische Bildlichkeit bei Hans Magnus Enzensberger. In: GRIMM, REINHARD (ed.): Texturen. Essays und anderes zu Hans Magnus Enzensberger. New York/Bern/Frankfurt (M.), 148-168.

KAISER, HERBERT (1993): Metapher Mensch. Zur Aktualität der offenen Anthropologie der deutschen Spätaufklärung. In: BORNSCHEUER, LOTHAR / KAISER, HERBERT / KulEnKAMPFF, Jens (eds.): Glaube - Kritik - Phantasie. Europäische Aufklärung in Religion und Politik, Wissenschaft und Literatur. Frankfurt (M.)/Berlin, 123-138.

- (1993a): Erfinden und Verschwinden. Über Phantasie, Tod und Metapher. In: LOQUAI, FrANZ (ed.): Gerhard Köpf. Eggingen, 173-184.

- (1995): Jean Paul lesen. Versuch über seine Anthropologie des Ich. Würzburg.

- (2005): Der Fotograf ist nicht im Bilde. Über Situation, Fantasie und Metapher. In: HeIMBÖCKel, DieTER / WeRLeIN, Uwe (eds.): Der Bildhunger der Literatur. Festschrift für Gunter E. Grimm. Würzburg, 27-36.

KoCH, MANFRED (1997): „Vor Neufundland vereinzelt Eisberge“. Zum Verhältnis von Utopie und Apokalypse in Enzensbergers „Untergang der Titanic“. In: JUNCKER, ROLF (ed.): Zeitgenössische Utopieentwürfe in Literatur und Gesellschaft. Amsterdam, 273-294.

KOENEN, GERD (2002): Das rote Jahrzehnt. Unsere kleine deutsche Kulturrevolution 1967-1977. Frankfurt (M.).

Komfort-Hein, Susanne (2001): Flaschenposten und kein Ende des Endes. 1968: Kritische Korrespondenzen um den Nullpunkt von Geschichte und Literatur. Freiburg. LAU, JÖRG (2001): Hans Magnus Enzensberger. Ein öffentliches Leben. Frankfurt (M.). LichtenBerg, GeORg Christoph (1968): Schriften und Briefe. Bd. 1: Sudelbücher 1. Hrsg. von Wolfgang Promies. München.

MANDELSTAM, OssiP (1985): Das Rauschen der Zeit. Gesammelte „autobiographische" Prosa der 20er Jahre. Hrsg. und übersetzt von Ralph Dutli. Zürich.

- (1994): Über den Gesprächspartner. Gesammelte Essays I (1913-1924). Hrsg. und übersetzt von Ralph Dutli. Frankfurt (M.).

- (1994a): Gespräch über Dante. Gesammelte Essays II (1925-1935). Hrsg. und übersetzt von Ralph Dutli. Frankfurt (M.). 
Zur Funktion der Metapher in Hans Magnus Enzensbergers „Der Untergang der Titanic“

SCHWEIKERT, RUdi (1985): Aus den „Erzählungen der tausendundein Nächte“. Karl Mays Anverwandlung einer Motivkombination in ,Scepter und Hammer': Enthaupten und Vergiften. In: Mitteilungen der Karl-May-Gesellschaft 63:3-7.

Thies-Lehmann, Hans (1984): Eisberg und Spiegelkunst. Notizen zu Hans Magnus Enzensbergers Lust am Untergang der Titanic (1978). In: GRIMM, 313-334.

Turgenjew, Iwan (1983): Tagebuch eines überflüssigen Menschen. Aus dem Russischen von Dieter Pommerenke. Berlin/Weimar. 